\title{
ON THE EULER CHARACTERISTIC OF FIBRES OF REAL POLYNOMIAL MAPS
}

\author{
ADAM PARUSIŃSKI \\ Departement de Mathématiques, Université d'Angers \\ 2, bd. Lavoisier, 49045 Angers Cedex 01, France \\ E-mail: parus@tonton.univ-angers.fr \\ ZBIGNIEW SZAFRANIEC \\ Institute of Mathematics, University of Gdańsk \\ Wita Stwosza 57, 80-952 Gdańsk, Poland \\ E-mail: szafran@ksinet.univ.gda.pl
}

\begin{abstract}
Let $Y$ be a real algebraic subset of $\mathbf{R}^{m}$ and $F: Y \rightarrow \mathbf{R}^{n}$ be a polynomial map. We show that there exist real polynomial functions $g_{1}, \ldots, g_{s}$ on $\mathbf{R}^{n}$ such that the Euler characteristic of fibres of $F$ is the sum of signs of $g_{i}$.

The purpose of this paper is to give a new, self-contained and elementary proof of the following result.

TheOREM. Let $Y$ be a real algebraic subset of $\mathbf{R}^{m}$ and $F: Y \rightarrow \mathbf{R}^{n}$ be a polynomial map. Then there exist real polynomials $g_{1}(y), \ldots, g_{s}(y)$ on $\mathbf{R}^{n}$ such that the Euler characteristic of fibres of $F$ is the sum of signs of $g_{i}$, that is

$$
\chi\left(F^{-1}(y)\right)=\operatorname{sgn} g_{1}(y)+\ldots+\operatorname{sgn} g_{s}(y) .
$$

Our proof is based on a classical and elementary result expressing the number of real roots of a real polynomial of one variable as the signature of an associated quadratic form known already to Hermite [He1, He2] and Sylvester [Syl], see also [B], [BW], [BCR, p. 97]. In the proof we use a modern generalized version of this result presented in [PRS] (note that we need only a one variable case of [PRS], that is precisely [BR, Proposition p. 18]). Our original proof of the theorem [PS] used different means such as the theory of local topological degree of polynomial mappings, Gröbner bases and the Eisenbud-Levine Theorem and was not so explicit as the one presented below.
\end{abstract}

1991 Mathematics Subject Classification: Primary 14P05, 14P25.

Received by the editors: January 23, 1997; in the revised form: November 15, 1997.

The paper is in final form and no version of it will be published elsewhere. 
The paper is organized as follows. In Section 1 we prepare the algebraic part of the proof. In particular we recall Hermite and Sylvester's theorem. In Section 2 we present basic properties of Euler characteristic of semialgebraic sets and a useful formalism of Euler integral of constructible functions. The proof of the theorem for $F$ proper is presented in Section 3. This case is particularly simple and the proof is obtained by an effective elimination procedure. In Section 4 we complete the proof in general (non-proper) case.

The main theorem of the paper was inspired by the first preprint version of $[\mathrm{CK}]$. For its applications see [MP] and [PS].

1. Preliminaries I. Let $W$ be an irreducible real algebraic subset of $\mathbf{R}^{n}$, let $\mathcal{A}$ denote the ring of real polynomials on $W$, and $\mathcal{K}$ - the field of fractions of $\mathcal{A}$.

Let $X$ be a real indeterminate. Take $h^{1}, \ldots, h^{k} \in \mathcal{K}[X]$ and denote by $I$ the ideal in $\mathcal{K}[X]$ generated by $h^{1}, \ldots, h^{k}$. We assume that at least one of $h^{i}$ is nonzero. The ring $\mathcal{K}[X]$ is a principal ideals domain, and $h=$ g.c.d. $\left(h^{1}, \ldots, h^{k}\right)$ is a generator of $I$. One may find polynomials $H_{1}, \ldots, H_{k}, P_{1}, \ldots, P_{k} \in \mathcal{K}[X]$ such that

$$
h=H_{1} h^{1}+\ldots+H_{k} h^{k}, \quad h^{i}=P_{i} h .
$$

Set $d=\operatorname{deg} h$. Then $Q=\mathcal{K}[X] / I$ is a $\mathcal{K}$-algebra and $d=\operatorname{dim}_{\mathcal{K}} Q$. The monomials $1, X, \ldots, X^{d-1}$ form a basis in $Q$. In particular, if $d=0$, then $I=\mathcal{K}[X]$ and $Q=\{0\}$.

For any $f \in \mathcal{K}[X]$ there are unique $q, r \in \mathcal{K}[X]$ such that $f=q h+r$ and $\operatorname{deg} r<d$. Thus $r=a_{0}+a_{1} X+\ldots+a_{d-1} X^{d-1}$. Put $r_{i}(f)=a_{i} \in \mathcal{K}$. This way $r_{i}(f)$ is the $i$-th coordinate of $f$ in $Q$.

Any $f \in Q$ defines a $\mathcal{K}$-linear endomorphism $A_{f}: Q \rightarrow Q$ by multiplication $A_{f}(p)=f p$. Let $\operatorname{Tr}(f) \in \mathcal{K}$ be the trace of $A_{f}$. Clearly

$$
\operatorname{Tr}(f)=r_{0}(f)+r_{1}(f X)+\ldots+r_{d-1}\left(f X^{d-1}\right) .
$$

The trace map $\operatorname{Tr}: Q \rightarrow \mathcal{K}$ is $\mathcal{K}$-linear.

Fix $g \in \mathcal{K}[X]$. Define a symmetric bilinear form $\Theta^{g}: Q \times Q \rightarrow \mathcal{K}$ by $\Theta^{g}(a, b)=\operatorname{Tr}(g a b)$. For $0 \leq i, j \leq d-1$ put $T^{i j}=r_{0}\left(g X^{i+j}\right)+\ldots+r_{d-1}\left(g X^{i+j+d-1}\right) \in \mathcal{K}$, so that the matrix of $\Theta^{g}$ is $\left[T^{i j}\right]$.

Recall that $\mathcal{K}$ is the field of fractions of the polynomial $\operatorname{ring} \mathcal{A}$ of an irreducible real algebraic set $W \subset \mathbf{R}^{n}$. There exists a proper algebraic subset $\Sigma \subset W$ such that the numerators and the denominators of all non-trivial elements of $\mathcal{K}$, which have appeared above, do not vanish on $W \backslash \Sigma$. Given $f \in \mathcal{K}[X], f=a_{d} X^{d}+\ldots+a_{0}, a_{i} \in \mathcal{K}$. For $w \in W$ we denote by $f_{w}$ the evaluation of $f$ at $w$, that is, $f_{w}=a_{d}(w) X^{d}+\ldots+a_{0}(w)$. Such $f_{w}$ is well-defined provided all $a_{i}(w)$ exist. Fix $w \in W \backslash \Sigma$. Then $\operatorname{deg} h_{w}=d$ and $h_{w}$ is the greatest common divisor of $h_{w}^{1}, \ldots, h_{w}^{k}$. Let $I_{w}$ denote the ideal generated by $h_{w}^{1}, \ldots, h_{w}^{k}$. Hence $I_{w}=\left(h_{w}\right), Q_{w}=\mathbf{R}[X] / I_{w}$ is an $\mathbf{R}$-algebra, $\operatorname{dim}_{\mathbf{R}} Q_{w}=d$, and $1, X, \ldots, X^{d-1}$ form a basis in $Q_{w}$.

Given $g \in \mathcal{K}[X]$. In the same way as above we define the trace map $\operatorname{Tr}_{w}: Q_{w} \rightarrow \mathbf{R}$, and the symmetric bilinear form $\Theta_{w}^{g}: Q_{w} \times Q_{w} \rightarrow \mathbf{R}$, given by $\Theta_{w}^{g}(a, b)=\operatorname{Tr}\left(g_{w} a b\right)$. Then the evaluation $\left[T_{w}^{i j}\right]=\left[T^{i j}(w)\right]$ is the matrix of $\Theta_{w}^{g}$.

Let $V_{w}=\left\{x \in \mathbf{R} \mid h^{1}(w, x)=\ldots=h^{k}(w, x)=0\right\}=\left\{x \in \mathbf{R} \mid h_{w}(x)=0\right\}$. If 
$w \in W \backslash \Sigma$ then $I_{w} \neq\{0\}$ and $V_{w}$ is finite. Set

$$
A_{w}=\sum_{x \in V_{w}} \operatorname{sgn} g(w, x) .
$$

If $g=1$ then $A_{w}$ is just the number of real roots of $h_{w}(x)$. The following result follows from [He1, He2] and [Syl], see [BR, Proposition p. 18] and [PRS, Theorem 2.1].

Proposition 1. For $w \in W \backslash \Sigma$

$$
A_{w}=\text { signature } \Theta_{w}^{g}=\operatorname{signature}\left[T_{w}^{i j}\right] .
$$

Now we apply the lemma of Descartes in order to describe the way $A_{w}$ depends on $w$.

LEMma 2. Let $f(x)=a_{d} x^{d}+a_{d-1} x^{d-1}+\ldots+a_{0}$ be a real polynomial and assume that all roots of $f(x)$ are real. Let $p_{+}$(resp. $p_{-}$) denote the number of positive (resp. negative) roots of $f$ counted with multiplicities. Let $\Lambda$ denote the set of all pairs $(r, s)$ with $0 \leq r<s \leq n$ such that $a_{r} \neq 0, a_{s} \neq 0$, and $a_{i}=0$ for $r<i<s$. Define $\Lambda^{\prime}=\{(r, s) \in \Lambda \mid r+s$ is odd $\}$. Then

$$
p_{+}-p_{-}=-\sum_{(r, s) \in \Lambda^{\prime}} \operatorname{sgn}\left(a_{r} a_{s}\right) .
$$

Proof. We say that the pair of real numbers $(a, b)$ changes sign if $a b<0$. If this is the case then $(1-\operatorname{sgn} a b) / 2=1$, if $a b>0$ then $(1-\operatorname{sgn} a b) / 2=0$.

By Descartes' lemma (see [MS, Theorem 6, p. 232], or [BR, Proposition 1.1.10, p. 14]), $p_{+}$equals the number of sign changes in the sequence of non-zero coefficients of $f(x)$, that is

$$
p_{+}=\sum_{(r, s) \in \Lambda}\left(1-\operatorname{sgn}\left(a_{r} a_{s}\right)\right) / 2 .
$$

According to the same fact, $p_{-}$equals the number of sign changes in the sequence of non-zero coefficients of $f(-x)$, i.e.

$$
p_{-}=\sum_{(r, s) \in \Lambda}\left(1-(-1)^{r+s} \operatorname{sgn}\left(a_{r} a_{s}\right)\right) / 2 .
$$

Hence $p_{+}-p_{-}=-\sum_{(r, s) \in \Lambda^{\prime}} \operatorname{sgn} a_{r} a_{s}$ as required.

Corollary 3. There exist polynomials $\varphi_{1}, \ldots, \varphi_{t} \in \mathcal{A}$ and a proper algebraic subset $\Sigma \subset W$ such that for every $w \in W \backslash \Sigma$

$$
A_{w}=\sum_{i=1}^{t} \operatorname{sgn} \varphi_{i}(w) .
$$

Proof. Let $T(\lambda)=T_{d} \lambda^{d}+\ldots+T_{0}$, where $T_{i} \in \mathcal{K}$, be the characteristic polynomial of $\left[T^{i j}\right]$. Define $\Lambda^{\prime}$ in the same way as above. In particular $T_{r} T_{s} \in \mathcal{K} \backslash\{0\}$ for each $(r, s) \in \Lambda^{\prime}$. Then the evaluation $T_{w}(\lambda)=T_{d}(w) \lambda^{d}+\ldots+T_{0}(w)$ is the characteristic polynomial of $\left[T_{w}^{i j}\right]$. We enlarge $\Sigma$ so that $T_{r}(w) T_{s}(w) \neq 0$ for $w \in W \backslash \Sigma$ and $(r, s) \in \Lambda^{\prime}$. Then by Lemma 2

$$
\text { signature } \Theta_{w}^{g}=\operatorname{signature}\left[T_{w}^{i j}\right]=-\sum_{(r, s) \in \Lambda^{\prime}} \operatorname{sgn} T_{r}(w) T_{s}(w)
$$

as required. 
2. Preliminaries II. In this section we recall the construction and some basic properties of the Euler integral of a constructible function, see e.g. [MP] for more information.

An integer-valued function $\varphi: \mathbf{R}^{n} \rightarrow \mathbf{Z}$ is called constructible if it admits a presentation as a finite sum

$$
\varphi=\sum m_{i} \mathbf{1}_{X_{i}}
$$

where for each $i, X_{i}$ is a closed semialgebraic subset of $\mathbf{R}^{n}, \mathbf{1}_{X_{i}}$ is the characteristic function of $X_{i}$, and $m_{i}$ 's are integers. The presentation (3) is not unique. If the support of $\varphi$ is compact, then we may choose all $X_{i}$ compact. Then the Euler integral of $\varphi$ is defined as

$$
\int \varphi=\sum m_{i} \chi\left(X_{i}\right)
$$

For $R>0$ let $\Psi_{R}^{n}$ denote the characteristic function of the ball $\mathbf{B}_{R}$ centred at the origin and of radius $R$. For any constructible $\varphi$, the product $\varphi \Psi_{R}^{n}$ has compact support, and we define the Euler integral of $\varphi$ as

$$
\int \varphi=\int \varphi \Psi_{R}^{n}, \quad \text { for } R \gg 0 .
$$

This definition makes sense because of the following

LEMma 4. Let $X \subset \mathbf{R}^{n}$ be semialgebraic. Then if $R>0$ is large enough, $X \cap \mathbf{B}_{R}$ is a deformation retract of $X$.

Proof. Let $\rho: X \rightarrow \mathbf{R}$ denote the distance to the origin. By topological triviality of semialgebraic mappings [BCR, Thm. 9.3.1], there is a finite subset $\left\{y_{1}, \ldots, y_{p}\right\} \subset \mathbf{R}$ such that

$$
\rho: X \backslash \rho^{-1}\left(\left\{y_{1}, \ldots, y_{p}\right\}\right) \rightarrow \mathbf{R} \backslash\left\{y_{1}, \ldots, y_{p}\right\}
$$

is a locally trivial fibration. In particular, $R>\max \left\{\left|y_{1}\right|, \ldots,\left|y_{p}\right|\right\}$ satisfies the statement of the lemma.

COROllary 5. If $X \subset \mathbf{R}^{n}$ is closed semialgebraic then $\int \mathbf{1}_{X}=\chi(X)$.

Proof. If $R$ is large enough then $X \cap \mathbf{B}_{R}$ is a deformation retract of $X$. Hence $\int \mathbf{1}_{X}=\int \mathbf{1}_{X} \Psi_{R}^{n}=\chi\left(X \cap \mathbf{B}_{R}\right)=\chi(X)$, as required.

Lemma 6. Let $f(x)=a_{d} x^{d}+\ldots+a_{0}, a_{d} \neq 0$, be a polynomial. Then $\varphi(x)=\operatorname{sgn} f(x)$ is a constructible function. For $2 \leq k \leq d$ define $V_{k}=\left\{x \in \mathbf{R} \mid f(x)=\ldots=f^{(k-1)}(x)=0\right\}$ and put $Z_{k}=\sum_{x \in V_{k}} \operatorname{sgn} f^{(k)}(x)$. Then

$$
\int \varphi= \begin{cases}\operatorname{sgn} a_{d}-\left(Z_{2}+Z_{4}+\ldots+Z_{d}\right), & \text { for d even } \\ -\left(Z_{2}+Z_{4}+\ldots+Z_{d-1}\right), & \text { for d odd }\end{cases}
$$

Proof. Clearly $\operatorname{sgn} f(x)$ is constructible. Let $x_{1}<\ldots<x_{p}$ be the real roots of $f$. Take $R>0$ such that $-R<x_{1}$ and $x_{p}<R$. Put $x_{0}=-R$ and $x_{p+1}=R$. For $0 \leq i \leq p+1$, let $k(i)=\min \left\{j \geq 0 \mid f^{(j)}\left(x_{i}\right) \neq 0\right\}$, and $S_{i}=\operatorname{sgn} f^{(k(i))}\left(x_{i}\right)$.

The sign of $f$ on $\left(x_{i}, x_{i+1}\right)$ is constant and equals $S_{i}=(-1)^{k(i+1)} S_{i+1}$. We have

$$
\varphi \psi_{R}^{1}=\sum_{i=0}^{p} \frac{1}{2}\left(S_{i}+(-1)^{k(i+1)} S_{i+1}\right) \mathbf{1}_{\left[x_{i}, x_{i+1}\right]}-\sum_{i=1}^{p}\left((-1)^{k(i)}+1\right) S_{i} \mathbf{1}_{\left\{x_{i}\right\}} .
$$


Hence

$$
\int \varphi=\frac{1}{2}\left(S_{0}+S_{p+1}\right)-\sum_{i=1}^{p} \frac{1}{2}\left((-1)^{k(i)}+1\right) S_{i}=\frac{1}{2}\left(S_{0}+S_{p+1}\right)-\left(Z_{2}+Z_{4}+\ldots\right) .
$$

If $d$ is even then $\frac{1}{2}\left(S_{0}+S_{p+1}\right)=\operatorname{sgn} a_{d}$. If $d$ is odd then $\frac{1}{2}\left(S_{0}+S_{p+1}\right)=0$. This ends the proof of Lemma 6 .

Proposition 7. Let $W$ be an irreducible real algebraic subset of $\mathbf{R}^{n}$ and $\mathcal{A}$ denote the ring of real polynomials on $W$. Let $f \in \mathcal{A}[X]$. Then there exist a finite family of polynomials $\psi_{i} \in \mathcal{A}$ and a proper algebraic subset $\Sigma \subset W$ such that for every $w \in W \backslash \Sigma$

$$
\int_{\mathbf{R}} \operatorname{sgn} f(w, \cdot)=\sum \operatorname{sgn} \psi_{i}(w) .
$$

Proof. Let $f=a_{r} x^{r}+\ldots+a_{0}$, where $a_{i} \in \mathcal{A}$. We may suppose $a_{r} \neq 0$ in $\mathcal{A}$. Then for $w \in W \backslash a_{r}^{-1}(0), \int_{\mathbf{R}} \operatorname{sgn} f(w, \cdot)$ is calculated in Lemma 6 .

For each $1 \leq k \leq r$, we apply the construction of Section 1 to the ideal $I=\left(h^{1}, \ldots, h^{k}\right)$ generated by $h^{1}=f, \ldots, h^{k}=\partial^{k-1} f / \partial X^{k-1}$ and $g=\partial^{k} f / \partial X^{k}$. Then, by Proposition 1 and Corollary 3 , each of $Z_{k}$ of Lemma 6 is a finite sum of signs of polynomials in $w$ as required. This shows the proposition.

Proposition 8. Let $W$ be a real algebraic set and $\mathcal{A}$ denote the ring of polynomials on $W$. Let $f \in \mathcal{A}[X]$. Then there exists a finite family of polynomials $\gamma_{i} \in \mathcal{A}$ such that for every $w \in W$

$$
\int_{\mathbf{R}} \operatorname{sgn} f(w, \cdot)=\sum_{i} \operatorname{sgn} \gamma_{i}(w) .
$$

Proof. The proof is by induction on $\operatorname{dim} W$ and the number of irreducible components of $W$.

If $W$ is irreducible then, by Proposition 7, we may find a finite family of polynomials $\gamma_{k}^{\prime} \in \mathcal{A}$ satisfying (4) in the complement of the proper algebraic subset $\Sigma$ of $W$. By the inductive assumption there exists a finite family of polynomials $\gamma_{j}^{\prime \prime}$ on $\Sigma$ satisfying (4) on $\Sigma$. We consider $\gamma_{j}^{\prime \prime}$ as the restriction of polynomials on $W$. Let $P \in \mathcal{A}$ be any nonnegative polynomial such that $P^{-1}(0)=\Sigma$. Then

$$
\operatorname{sgn} P \gamma_{k}^{\prime}= \begin{cases}\operatorname{sgn} \gamma_{k}^{\prime}, & \text { on } W \backslash \Sigma ; \\ 0, & \text { on } \Sigma\end{cases}
$$

Similarly

$$
\operatorname{sgn} \gamma_{j}^{\prime \prime}-\operatorname{sgn} P \gamma_{j}^{\prime \prime}= \begin{cases}0, & \text { on } W \backslash \Sigma ; \\ \operatorname{sgn} \gamma_{j}^{\prime \prime}, & \text { on } \Sigma,\end{cases}
$$

and hence the family $\left\{P \gamma_{k}^{\prime}, \gamma_{j}^{\prime \prime},-P \gamma_{j}^{\prime \prime}\right\}$ satisfies the statement.

Suppose $W^{\prime} \subset W$ is an irreducible component of $W$ and let $W^{\prime \prime}$ be the union of the other components. Let $\gamma_{k}^{\prime}$ (resp. $\gamma_{j}^{\prime \prime}$ ) be the family satisfying (4) on $W^{\prime}$ (resp. $W^{\prime \prime}$ ). Let $P \in \mathcal{A}$ be any non-negative polynomial such that $P^{-1}(0)=W^{\prime}$. Then, by the same argument as above, the family $\left\{P \gamma_{k}^{\prime}, \gamma_{j}^{\prime \prime},-P \gamma_{j}^{\prime \prime}\right\}$ satisfies the statement. This ends the proof. 
3. Proof of Theorem (proper case). Suppose $F: Y \rightarrow \mathbf{R}^{n}$ is proper. Replacing $Y$ by the graph of $F$ we may assume that $Y \subset \mathbf{R}^{n} \times \mathbf{R}^{m}$ and $F$ is induced by the projection on the first factor.

The proof is by induction on $m$ (we do not assume $n$ to be fixed). Suppose $m=1$ and $Y$ is the zero set of a non-negative polynomial function $f(y, x)$. We apply Proposition 8 to $W=\mathbf{R}^{n}$ and $\mathcal{A}=\mathbf{R}[y]$. Note that, since $F$ is proper, $F^{-1}(y)$ has to be finite and $\chi\left(F^{-1}(y)\right)=1-\int_{\mathbf{R}} \operatorname{sgn} f(y, \cdot)$. Hence the result follows from Proposition 8.

Inductive step. Let $p: Y \rightarrow \mathbf{R}^{n} \times \mathbf{R}^{1}$ denote the projection. By the inductive hypothesis we may assume that there exists a finite family of polynomials $f_{i}\left(y, x_{1}\right)$ such that for each $\left(y, x_{1}\right) \in \mathbf{R}^{n} \times \mathbf{R}$

$$
\chi\left(p^{-1}\left(\left(y, x_{1}\right)\right)\right)=\sum_{i} \operatorname{sgn} f_{i} .
$$

Define $\varphi\left(y, x_{1}\right)=\chi\left(p^{-1}\left(\left(y, x_{1}\right)\right)\right.$. We claim that

$$
\int_{\mathbf{R}} \varphi(y, \cdot)=\chi\left(F^{-1}(y)\right) \text {. }
$$

Indeed, this follows from a "Fubini-type" formula for the Euler integral,

$$
\int_{\{y\} \times \mathbf{R}} \varphi(y, \cdot)=\int_{\{y\} \times \mathbf{R}} p_{*} \mathbf{1}_{Y}=\int_{F^{-1}(y)} \mathbf{1}_{Y}=\chi\left(F^{-1}(y)\right),
$$

see, e.g., [V], [MP, A.4.2]. Then, by (5) and (6)

$$
\chi\left(F^{-1}(y)\right)=\sum_{i} \int_{\mathbf{R}} \operatorname{sgn} f_{i}(y, \cdot),
$$

and the theorem again follows from Proposition 8 .

4. Proof of Theorem (general case). The proof presented in the previous sections does not work in general since the "Fubini type" formula (7) fails for non-proper maps. To complete the proof in the general case we use the projective compactification and the link at infinity. We shall also need the following corollary of the theorem.

Proposition 9. Let $Y \subset \mathbf{R}^{m}$ be algebraic and $F: Y \rightarrow \mathbf{R} \times \mathbf{R}^{n}$ be a proper polynomial map. Then there exists a finite family of polynomials $\gamma_{i}(y)$, where $y \in \mathbf{R}^{n}$, such that

$$
\lim _{t \rightarrow 0^{+}} \chi\left(F^{-1}(t, y)\right)=\sum \operatorname{sgn} \gamma_{i}(y) .
$$

Pr o of. The proposition follows easily from the theorem and the following lemma.

LEMma 10. Let $W$ be real algebraic and $g(t, w)$ be a polynomial on $\mathbf{R} \times W$. Then there exists a finite family of polynomials $\gamma_{i}(w)$ on $W$ such that

$$
\lim _{t \rightarrow 0^{+}} \operatorname{sgn} g(t, w)=\sum \operatorname{sgn} \gamma_{i}(w)
$$

Proof. Let $\psi(w)=\lim _{t \rightarrow 0^{+}} g(t, w)$. We proceed by induction on $\operatorname{dim} W$. Without loss of generality we may assume that $W$ is irreducible. We shall show that the statement of lemma holds generically on $W$, that is to say, there exist a proper algebraic subset $W^{\prime}$ of $W$ and the polynomials $\gamma_{i}$ such that (9) holds in the complement of $W^{\prime}$. Then the lemma will follow from the inductive assumption. 
We may also assume that $g$ does not vanish identically, and then there exists a nonnegative integer $k$ such that $g(t, w)=t^{k} h(t, w)$, where $h(t, w)$ is a polynomial which does not vanish identically on $\{0\} \times W$. Then, in the complement of $W^{\prime}=\{w \mid h(0, w)=0\}$, $\psi(w)=\operatorname{sgn} h(0, w)$ as required. This ends the proof of the lemma and the proposition.

Remark 11. The assumption of properness of $F$ in Proposition 9 is not necessary. We made it since we are going to use Proposition 9 in the proof of theorem in general case, and it is only the proper case of theorem which has been proven till now.

Let $X$ be a real algebraic subset of $\mathbf{R}^{m}$ and denote by $\widetilde{X}$ the one point compactification of $X$ (if $X$ is compact then $\widetilde{X}=X \amalg$ ppoint $\}$ ). Let $L_{R}=X \cap S_{R}^{m-1}$, where $S_{R}^{m-1}$ denotes the sphere of radius $R>0$ centred at the origin. We call such $L_{R}$, for $R$ sufficiently large, the link at infinity of $X$, and denote by $L_{\infty}(X)$. By an argument similar to the proof of Lemma 4 it is easy to see that

$$
\chi(X)=\chi(\widetilde{X})+\chi\left(L_{\infty}(X)\right)-1
$$

Let us recall the standard construction of $\tilde{X}$ as an algebraic set (we will need it below in a parametrized case). Suppose $X$ is given by a finite number of equations $f_{i}(x)=0$, $i=1, \ldots, s$. Set $h\left(x, x_{m+1}\right)=\left(f_{1}^{2}(x)+\ldots+f_{s}^{2}(x)\right)+\left(x_{m+1}-1\right)^{2}$, so that $h^{-1}(0)$ is homeomorphic to $X$ and $h$ is a non-negative polynomial of degree, say, bounded by $2 p$. Put $x^{\prime}=\left(x, x_{m+1}\right) \in \mathbf{R}^{m+1}$ and let $H\left(s, x^{\prime}\right)=\left(s\left\|x^{\prime}\right\|\right)^{4 p} h\left(x^{\prime} /\left(s\left\|x^{\prime}\right\|\right)^{2}\right)$. Then, it is easy to see that $H$ extends to a non-negative polynomial on $\mathbf{R} \times \mathbf{R}^{m+1}$ such that for $s \neq 0$, $\widetilde{X}_{s}=\left\{\left(x^{\prime} \in \mathbf{R}^{m+1} \mid H\left(s, x^{\prime}\right)=0\right\}\right.$ is homeomorphic to the single point compactification of $X$. Also $L_{s}=\left\{\left(x^{\prime} \in \mathbf{R}^{m+1} \mid H\left(s, x^{\prime}\right)=0,\left\|x^{\prime}\right\|=1\right\}\right.$, for $s \neq 0$ and small enough, is homeomorphic to the link at infinity of $X$.

Now we are ready to prove the theorem. First we assume that $Y \subset \mathbf{R}^{n} \times \mathbf{R}^{m}$ and $F$ is induced by the projection onto the first factor. Proceeding exactly in the same way as above we may compactify simultaneously the fibres of $F$. In particular the following statement holds.

Proposition 12. Let $Y \subset \mathbf{R}^{n} \times \mathbf{R}^{m}$ be algebraic and $F: Y \rightarrow \mathbf{R}^{n}$ be the projection on the first factor. Then there exists a non-negative polynomial $H: \mathbf{R} \times \mathbf{R}^{n} \times \mathbf{R}^{m+1} \rightarrow \mathbf{R}$ such that for every $y \in \mathbf{R}^{n}$

(i) $\tilde{Y}=\left\{x^{\prime} \in \mathbf{R}^{m+1} \mid H\left(s, y, x^{\prime}\right)=0, s=1\right\}$ is homeomorphic to the single point compactification of $F^{-1}(y)$. Moreover, the induced projection $\widetilde{Y} \rightarrow \mathbf{R}^{n}$ is proper;

(ii) $\left\{x^{\prime} \in \mathbf{R}^{m+1} \mid H\left(s, y, x^{\prime}\right)=0,\left\|x^{\prime}\right\|=1\right\}$, for $s=s(y) \neq 0$ small enough, is homeomorphic to the link at infinity of $F^{-1}(y)$.

Note that identity (10) holds for every $X=F^{-1}(y)$. Hence to prove the theorem it suffices to consider the families of compactifications and links at infinity of fibres $F^{-1}(y)$ parametrized by $y \in \mathbf{R}^{n}$. For the first one the statement holds by Proposition 12 (i) and the proper case. For the family of links consider $L \subset \mathbf{R} \times \mathbf{R}^{n} \times \mathbf{R}^{m+1}$ given by $H\left(s, y, x^{\prime}\right)=0,\left\|x^{\prime}\right\|=1$. Then, clearly the projection $L \rightarrow \mathbf{R} \times \mathbf{R}^{n},\left(s, y, x^{\prime}\right) \rightarrow(s, y)$, is proper and we apply to it Proposition 9. Now Proposition 12 (ii) gives the statement for the family of links at infinity. This, in virtue of (10), shows the theorem. 


\section{References}

[B] E. Becker, Sums of squares and trace forms in real algebraic geometry, in: De la géométrie algébrique réelle (Paris, 1990), Cahiers Sém. Hist. Math. Sér. 2 vol. 1, Université Pierre et Marie Curie, Paris, 1991, 41-57.

[BW] E. Becker, T. Wöermann, On the trace formula for quadratic forms and some applications, in: Recent Advances in Real Algebraic Geometry and Quadratic Forms, Contemp. Math. 155, Amer. Math. Soc., Providence, 1994, 271-291.

[BR] R. Benedetti, J.-J. Risler, Real Algebraic and Semi-algebraic Sets, Actualités Math., Hermann, Paris, 1990.

[BCR] J. Bochnak, M. Coste, M.-F. Roy, Géométrie algébrique réelle, Ergeb. Math. Grenzgeb. (3) 12, Springer, Berlin, 1987.

[CK] M. Coste, K. Kurdyka, Le discriminant d'un morphisme de variétés algébriques réelles, Topology 37 (1998), 393-400.

[He1] C. Hermite, Remarques sur le théorème de Sturm, C. R. Acad. Sci. Paris 36 (1853), $52-54$.

[He2] C. Hermite, Sur l'extension du théorème de M. Sturm à un système d'équations simultanées, Oeuvres de Charles Hermite, Tome 3, ed. E. Picard, Edition Paris, GauthierVillars, 1912, 1-34.

[MP] C. McCrory, A. Parusiński, Algebraically constructible functions, Ann. Scient. École Norm. Sup. (4) 30 (1997), 527-552.

[MS] A. Mostowski, M. Stark, Elementy algebry wyższej, Państwowe Wydawnictwo Naukowe, Warszawa, 1963 (in Polish); English translation: Introduction to Higher Algebra, Internat. Series of Monographs on Pure and Appl. Math. 37, A Pergamon Press Book, New York, and Państwowe Wydawnictwo Naukowe, Warszawa, 1964.

[PS] A. Parusiński, Z. Szafraniec, Algebraically constructible functions and signs of polynomials, Manuscripta Math. 93 (1997), 443-456.

[PRS] P. Pedersen, M.-F. Roy, A. Szpirglas, Counting real zeros in the multivariate case, in: Computational Algebraic Geometry, F. Eyssette, A. Galligo (eds.), Progr. Math. 109, Birkhäuser, Boston, 1993, 203-223.

[Syl] J. J. Sylvester, On a theory of syzygetic relations of two rational integral functions, comprising an application to the theory of Sturm's functions, Philos. Trans. Roy. Soc. London 143 (1853).

[V] O. Y. Viro, Some integral calculus based on Euler characteristic, in: Topology and Geometry-Rohlin Seminar, O. Y. Viro (ed.), Lecture Notes in Math. 1346, Springer, Berlin, 1988, 127-138. 